\title{
ON INTERVAL VALUED FUZZY $h$-IDEALS IN HEMIRINGS
}

\author{
Muhammad Shabir, Noshin Malik, and Tahir Mahmood
}

\begin{abstract}
In this paper we discuss some results associated with interval valued fuzzy $h$-ideals of hemirings and characterize hemirings by the properties of their interval valued fuzzy $h$-ideals.
\end{abstract}

\section{Introduction}

Semirings which are regarded as generalizations of associative rings were first introduced by Vandiver [23] in 1934. In more recent times semirings have been deeply studied, especially in relation with applications [8]. Semirings have also been used for studying optimization, graph theory, theory of discrete event dynamical systems, matrices, determinants, generalized fuzzy computation, theory of automata, formal language theory, coding theory, analysis of computer programmes $[4,5,8,9,20]$. Additively commutative semirings with zero element are called hemirings. Hemirings, appears in a natural manner, in some applications to the theory of automata, the theory of formal languages and in computer sciences $[10,11,16]$. Ideals of hemirings and semirings play an important role in the structure theory and are very useful for many purposes. However, in general, they do not coincide with the usual ring ideals. Many results in rings apparantly have no analogues in hemirings and semirings using only ideals. In order to overcome this difficulty in [12] Henriksen defined a more restricted class of ideals in semirings, called $k$-ideals, with the property that if the semiring $R$ is the ring, then a complex in $R$ is a $k$-ideal if and only if it is a ring ideal. Another more restricted, but very important, class of ideals in hemirings, called now $h$-ideals, has been given and investigated by Iizuka [13] and La Torre [14]. $h$-ideals are also discussed in [7]. In 1965 Zadeh [24] introduced the concept of fuzzy sets. Since then fuzzy sets has been applied to many branches in Mathematics. The fuzzification of algebraic structures was initiated by Rosenfeld [17] and he introduced the notion of fuzzy subgroups. In [2] J. Ahsan initiated the study of fuzzy semirings (See also [3]). The fuzzy algebraic structures play an important role in Mathematics with wide applications in many other branches such as theoretical physics, computer sciences,

Received April 28, 2011; Accepted August 7, 2011.

2000 Mathematics Subject Classification. 16Y60, 08A72, 03G25, 03E72.

Key words and phrases. Hemirings, fuzzy $h$-ideals, interval valued fuzzy $h$-ideals, $h$ hemiregular. 
control engineering, information sciences, coding theory and topological spaces $[1,10,20]$. The concept of interval valued fuzzy sets in algebra was initiated in [6] by Biswas and further this concept was investigated in [15]. In [21] Xueling and Zhan defined interval valued fuzzy $h$-ideals of hemirings and discussed some results associated with interval valued fuzzy $h$-ideals of hemirings. This concept is further carried out in [18]. In this paper we discuss some other results associated with interval valued fuzzy $h$-ideals of hemirings and characterize hemirings by the properties of their interval valued fuzzy $h$-ideals.

\section{Preliminaries}

A non-empty set $R$ together with two associative binary operations "+" and "." is said to be semiring if distributive laws hold in $R$. An element $0 \in R$ satisfying the conditions, $0 x=x 0=0$ and $0+x=x+0=x$, for all $x \in R$, is called zero of the semiring $(R,+, \cdot)$. An element $1 \in R$ satisfying the condition, $1 . x=x .1=x$ for all $x \in R$, is called identity of the semiring $R$. A semiring with commutative multiplication is called a commutative semiring. A semiring with commutative addition and zero element is called a hemiring. A non-empty subset $A$ of $R$ is called a subhemiring of $R$ if it contains zero and is closed with respect to the addition and multiplication of $R$. An element $a$ of $R$ is called multiplicatively idempotent if $a^{2}=a$. A hemiring $R$ is called multiplicatively idempotent if each element of $R$ is multiplicatively idempotent. A non-empty subset $A$ of $R$ is called a left (right) ideal of $R$ if $A$ is closed under addition and $R A \subseteq A(A R \subseteq A)$. If $A$ and $B$ are left (respectively right) ideals of a hemiring $R$ then $A \cap B$ is a left (respectively right) ideal of $R$. If $A$ is a subset of $R$, then intersection of all left (right) ideals of $R$ which contain $A$ is a left (right) ideal of $R$ containing $A$. Of course this is the smallest left (right) ideal of $R$ containing $A$ and is called the left (right) ideal of $R$ generated by $A$. If $A$ and $B$ are left (respectively right) ideals of a hemiring $R$ then $A+B$ is the smallest left (respectively right) ideal of $R$ containing both $A$ and $B$. If $A$ and $B$ are ideals of a hemiring $R$ then $A B$ is an ideal of $R$ contained in $A \cap B$. An ideal $A$ (left, right or two-sided) of a hemiring $R$ is called a $k$-ideal of $R$ if for any $y, z \in A$ and $x \in R$, from $x+y=z$ it follows that $x \in A$. An ideal $A$ (left, right or two-sided) of a hemiring $R$ is called an $h$-ideal of $R$ if for any $a, b \in A$ and $x, y \in R$, from $x+a+y=b+y$, it follows that $x \in A$. Clearly, a left (respectively right) $h$-ideal is always a left (respectively right) $k$-ideal but the converse is not always true. A fuzzy subset $f$ of a universe $X$ is a function $\lambda: X \longrightarrow[0,1]$. Furthermore for $t \in[0,1]$, level subset of $\lambda$ is denoted and defined by $U(\lambda, t)=\{x \in R: \lambda(x) \geq t\}$. For any two fuzzy subsets $\lambda$ and $\mu$ of $X, \lambda \leq \mu$ means that, for all $x \in X, \lambda(x) \leq \mu(x)$. The symbols $\lambda \wedge \mu$, and $\lambda \vee \mu$ will mean the following fuzzy subsets of $X$

$$
\begin{aligned}
& (\lambda \wedge \mu)(x)=\lambda(x) \wedge \mu(x), \\
& (\lambda \vee \mu)(x)=\lambda(x) \vee \mu(x)
\end{aligned}
$$


for all $x \in X$. More generally, if $\left\{\lambda_{i}: i \in \Lambda\right\}$ is a family of fuzzy subsets of $X$, then $\wedge_{i \in \Lambda} \lambda_{i}$ and $\vee_{i \in \Lambda} \lambda_{i}$ are defined by

$$
\begin{aligned}
& \left(\wedge_{i \in \Lambda} \lambda_{i}\right)(x)=\wedge_{i \in \Lambda}\left(\lambda_{i}(x)\right), \\
& \left(\vee_{i \in \Lambda} \lambda_{i}\right)(x)=\vee_{i \in \Lambda}\left(\lambda_{i}(x)\right) .
\end{aligned}
$$

Definition 1. Let $\lambda$ and $\mu$ be any two fuzzy subsets of a hemiring $R$. Then

(i) the sum of $\lambda$ and $\mu$ is defined as

$$
(\lambda+\mu)(x)=\vee_{x=y+z}[\lambda(y) \wedge \mu(z)] \quad \text { for all } x \in R .
$$

(ii) the product of $\lambda$ and $\mu$ is defined as

$(\lambda \mu)(x)$

$=\left\{\begin{array}{l}\vee_{x=\Sigma_{i=1}^{n} y_{i} z_{i}}\left[\wedge_{i}\left\{\lambda\left(y_{i}\right) \wedge \mu\left(z_{i}\right)\right\}\right] \text { if } x \text { can be expressed as } x=\sum_{i=1}^{n} y_{i} z_{i} \\ 0\end{array}\right.$

Definition 2. ([21]) The $h$-closure $\bar{A}$ of a non-empty subset $A$ of a hemiring $R$ is defined as

$$
\bar{A}=\{x \in R \mid x+a+z=b+z \text { for some } a, b \in A, z \in R\} .
$$

Definition 3. ([21]) Let $\lambda$ and $\mu$ be two fuzzy subsets in a hemiring $R$. The h-intrinsic product of $\lambda$ and $\mu$ is defined by

$$
(\lambda \odot \mu)(x)=\left\{\begin{array}{rr}
\vee_{x+\sum_{i=1}^{n} a_{i} b_{i}+z=\Sigma_{j=1}^{m} c_{j} d_{j}+z}\left\{\wedge _ { i , j } \left[\lambda\left(a_{i}\right) \wedge \lambda\left(c_{j}\right) \wedge\right.\right. \\
\left.\mu\left(b_{i}\right) \wedge \mu\left(d_{j}\right)\right\} \text { if } x \text { can be expressed as } \\
x+\Sigma_{i=1}^{n} a_{i} b_{i}+z=\Sigma_{j=1}^{m} c_{j} d_{j}+z \\
0 \quad \text { otherwiser }
\end{array}\right.
$$

Lemma 2.1. ([21]) Let $R$ be a hemiring and $A, B \subseteq R$. Then

(i) $\bar{A}$ is the smallest left $h$-ideal of $R$ containing $A$,

(ii) $\overline{(\bar{A})}=\bar{A}$,

(iii) If $A \subseteq B$, then $\bar{A} \subseteq \bar{B}$,

(iv) $A \subseteq B$ if and only if $C_{A} \leq C_{B}$,

(v) $C_{A} \wedge C_{B}=C_{A \cap B}$,

(vi) $C_{A} \odot C_{B}=C_{\overline{A B}}$,

(vii) $\overline{A B}=\overline{A B}$.

Definition 4. A fuzzy subset $\lambda$ of a hemiring $R$ is said to be a fuzzy left (respectively right) ideal of the hemiring $R$ if for all $x, y \in R$

(i) $\lambda(x+y) \geq \lambda(x) \wedge \lambda(y)$,

(ii) $\lambda(x y) \geq \lambda(y)$ (respectively $\lambda(x y) \geq \lambda(x)$ ).

A fuzzy subset $\lambda$ of a hemiring $R$ is called a fuzzy ideal of hemiring $R$ if it is both, fuzzy left and right ideal of $R$.

Theorem 2.2. If $\lambda$ and $\mu$ are fuzzy left (respectively right) ideals of a hemiring $R$ then $\lambda \wedge \mu$ is also a fuzzy left (respectively right) ideal of $R$. 
Lemma 2.3. ([22]) If $I$ and $L$ are respectively, right and left $h$-ideals of $a$ hemiring $R$, then $\overline{I L} \subseteq I \cap L$.

Definition 5. ([21]) A hemiring $R$ is said to be h-hemiregular if for each $x \in R$, there exist $a, b, z \in R$ such that $x+x a x+z=x b x+z$.

Lemma 2.4. ([21]) A hemiring $R$ is h-hemiregular if and only if for any right $h$-ideal $I$ and any left $h$-ideal $L$ of $R$ we have $\overline{I L}=I \cap L$.

Theorem 2.5. ([22]) A hemiring $R$ is h-hemiregular if and only if for any fuzzy right h-ideal $\lambda$ and any fuzzy left h-ideal $\mu$ of $R$ we have $\lambda \odot \mu=\lambda \wedge \mu$.

Let $£$ be the family of all closed subintervals of $[0,1]$. Define order $" \leq$ " on $£$ as:

$\left[\alpha, \alpha^{\prime}\right] \leq\left[\beta, \beta^{\prime}\right]$ if and only if $\alpha \leq \beta, \alpha^{\prime} \leq \beta^{\prime}$, for all $\left[\alpha, \alpha^{\prime}\right],\left[\beta, \beta^{\prime}\right] \in £$.

Then " $\leq$ " is partial order on $£$ with minimal element $\tilde{O}=[0,0]$ and maximal element $\tilde{I}=[1,1]$.

Definition 6. An interval valued fuzzy subset $\lambda$ of a hemiring $R$ is a function $\lambda: R \rightarrow £$.

We write $\lambda(x)=\left[\lambda^{-}(x), \lambda^{+}(x)\right] \subseteq[0,1]$, for all $x \in R$. Where $\lambda^{-}, \lambda^{+}$: $R \rightarrow[0,1]$ are lower and upper fuzzy sets of $R$, giving lower and upper limit of the image interval for each $x \in R$. Note that we have $0 \leq \lambda^{-}(x) \leq 1$ and $0 \leq \lambda^{+}(x) \leq 1$ for all $x \in R$. For simplicity we write $\lambda=\left[\lambda^{-}, \lambda^{+}\right]$.

Definition 7. For any two interval valued fuzzy subsets $\tilde{\lambda}$ and $\tilde{\mu}$ of a hemiring $R$, union and intersection are defined, for all $x \in R$

$$
\begin{aligned}
& (\tilde{\lambda} \vee \tilde{\mu})(\times)=\left[\lambda^{-}(x) \vee \tilde{\mu}^{-}(x), \lambda^{+}(x) \vee \tilde{\mu}^{+}(x)\right] \\
& (\tilde{\lambda} \wedge \tilde{\mu})(\times)=\left[\lambda^{-}(x) \wedge \tilde{\mu}^{-}(x), \lambda^{+}(x) \wedge \tilde{\mu}^{+}(x)\right] .
\end{aligned}
$$

More generally if $\left\{\tilde{\lambda}_{i}: i \in I\right\}$ is a family of interval valued fuzzy subsets of $R$ then for all $x \in R$,

$$
\begin{aligned}
& \left(\vee_{i} \tilde{\lambda}_{i}\right)(x)=\left[\vee_{i} \lambda_{i}^{-}(x), \vee_{i} \lambda_{i}^{+}(x)\right] \\
& \left(\wedge_{i} \tilde{\lambda}_{i}\right)(x)=\left[\wedge_{i} \lambda_{i}^{-}(x), \wedge_{i} \lambda_{i}^{+}(x)\right] .
\end{aligned}
$$

Definition 8. Let $\tilde{\lambda}$ and $\tilde{\mu}$ be interval valued fuzzy subsets of a hemiring $R$,

(1) then their sum is defined as

$(\tilde{\lambda}+\tilde{\mu})(x)=\vee_{x=y+z}\left[\lambda^{-}(y) \wedge \mu^{-}(z), \lambda^{+}(y) \wedge \mu^{+}(z)\right] \quad$ for all $x \in R$.

(2) then their product is defined as

$$
(\tilde{\lambda} \tilde{\mu})(x)=\left\{\begin{array}{r}
\vee_{x=\Sigma_{i=1}^{n} y_{i} z_{i}}\left\{\wedge_{i}\left[\begin{array}{r}
\lambda^{-}\left(y_{i}\right) \\
\lambda^{+}\left(y_{i}\right) \wedge \mu^{-}\left(z_{i}\right), \\
\text { if } x \text { can be expressed as } x=\sum_{i=1}^{n} y_{i} z_{i}
\end{array}\right]\right\} \\
\tilde{O} \quad \text { otherwise. }
\end{array}\right.
$$


Definition 9. An interval valued fuzzy subset $\tilde{\lambda}$ of a hemiring $R$ is said to be an interval valued fuzzy left (respectively right) ideal of the hemiring $R$ if for all $x, y \in R$

(i) $\tilde{\lambda}(x+y) \geq \tilde{\lambda}(x) \wedge \tilde{\lambda}(y)$,

(ii) $\tilde{\lambda}(x y) \geq \tilde{\lambda}(y)($ respectively $\tilde{\lambda}(x y) \geq \tilde{\lambda}(x))$.

An interval valued fuzzy subset $\tilde{\lambda}$ of a hemiring $R$ is called an interval valued fuzzy ideal of the hemiring $R$ if it is both interval valued fuzzy left and interval valued fuzzy right ideal of $R$.

Definition 10. Let $A$ be a subset of a hemiring $R$. Then the interval valued characteristic function $\tilde{C}_{A}$ of $A$ is defined to be a function $\tilde{C}_{A}: R \rightarrow £$ such that for all $x \in R$

$$
\tilde{C}_{A}(x)= \begin{cases}\tilde{I} & \text { if } x \in A \\ \tilde{O} & \text { if } x \notin A .\end{cases}
$$

Clearly the interval valued characteristic function of any subset of $R$ is also an interval valued fuzzy subset of $R$. The interval valued characteristic function can be used to indicate either membership or non-membership of any member of $R$ in a subset $A$ of $R$. Note that $\tilde{C}_{R}(x)=\tilde{I}$ for all $x \in R$.

Definition 11. ([19]) Let $\tilde{\lambda}$ and $\tilde{\mu}$ be interval valued fuzzy subsets of a hemiring $R$. Then their $h$-intrinsic product is denoted and defined by

$$
\begin{aligned}
& (\tilde{\lambda} \odot \tilde{\mu})(x)
\end{aligned}
$$

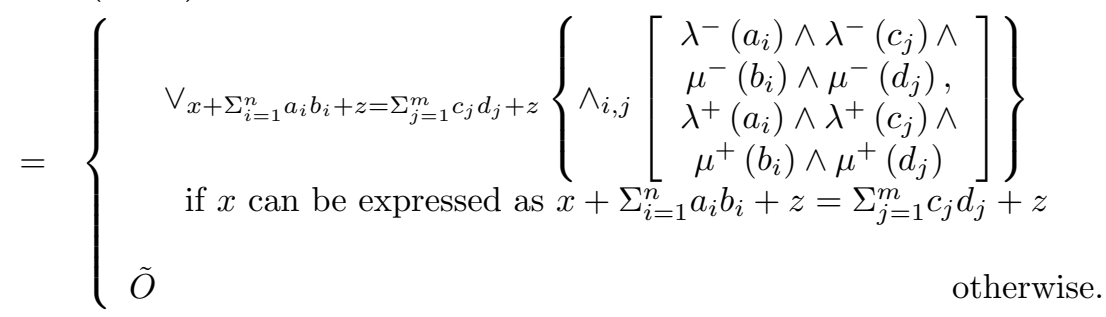

Lemma 2.6. ([19])

Let $R$ be a hemiring and $A, B \subseteq R$. Then

(i) $A \subseteq B$ if and only if $\tilde{C}_{A} \leq \tilde{C}_{B}$,

(ii) $\tilde{C}_{A} \wedge \tilde{C}_{B}=\tilde{C}_{A \cap B}$,

(iii) $\tilde{C}_{A} \odot \tilde{C}_{B}=\tilde{C}_{\overline{A B}}$.

Definition 12. An interval valued fuzzy left (resp. right) ideal $\tilde{\lambda}$ of a hemiring $R$ is called an interval valued fuzzy left (resp. right) $k$-ideal of $R$ if for all $x, y, z \in R$,

$$
x+y=z \Rightarrow \tilde{\lambda}(x) \geq \tilde{\lambda}(y) \wedge \tilde{\lambda}(z) .
$$

Definition 13. An interval valued fuzzy left (resp.right) ideal $\tilde{\lambda}$ of a hemiring $R$ is called an interval valued fuzzy left (resp. right) $h$-ideal of $R$ if for all $a, b, x, y \in R, x+a+y=b+y \Rightarrow \tilde{\lambda}(x) \geq \tilde{\lambda}(a) \wedge \tilde{\lambda}(b)$. 
Lemma 2.7. ([21]) A subset $A$ of a hemiring $R$ is an $h$-(resp. $k$-) ideal of $R$ if and only if the interval valued characteristic function $\tilde{C}_{A}$ is an interval valued fuzzy $h$-(resp. $k$-) ideal of $R$.

Remark 1. Every interval valued fuzzy $h$-ideal is an interval valued fuzzy left $k$-ideal but the converse is not true.

Example 1. Consider the semiring $R=\{0,1, a, b, c\}$ defined by the following tables:

\begin{tabular}{c|ccccc}
+ & 0 & 1 & $\mathrm{a}$ & $\mathrm{b}$ & $\mathrm{c}$ \\
\hline 0 & 0 & 1 & $\mathrm{a}$ & $\mathrm{b}$ & $\mathrm{c}$ \\
1 & 1 & $\mathrm{~b}$ & 1 & $\mathrm{a}$ & 1 \\
$\mathrm{a}$ & $\mathrm{a}$ & 1 & $\mathrm{a}$ & $\mathrm{b}$ & $\mathrm{a}$ \\
$\mathrm{b}$ & $\mathrm{b}$ & $\mathrm{a}$ & $\mathrm{b}$ & 1 & $\mathrm{~b}$ \\
$\mathrm{c}$ & $\mathrm{c}$ & 1 & $\mathrm{a}$ & $\mathrm{b}$ & $\mathrm{c}$
\end{tabular}

\begin{tabular}{c|ccccc}
$\cdot$ & 0 & 1 & $\mathrm{a}$ & $\mathrm{b}$ & $\mathrm{c}$ \\
\hline 0 & 0 & 0 & 0 & 0 & 0 \\
1 & 0 & 1 & $\mathrm{a}$ & $\mathrm{b}$ & $\mathrm{c}$ \\
$\mathrm{a}$ & 0 & $\mathrm{a}$ & $\mathrm{a}$ & $\mathrm{a}$ & $\mathrm{c}$ \\
$\mathrm{b}$ & 0 & $\mathrm{~b}$ & $\mathrm{a}$ & 1 & $\mathrm{c}$ \\
$\mathrm{c}$ & 0 & $\mathrm{c}$ & $\mathrm{c}$ & $\mathrm{c}$ & 0
\end{tabular}

Then the ideals of $R$ are $\{0\},\{0, c\},\{0, a, c\}$ and $R . A=\{0, c\}$ is $k$-ideal of $R$ but not an $h$-ideal of $R$, because $a+c+b=0+b$, but $a \notin A$. Then by Lemma 2.7, $\tilde{C}_{A}$ is an interval valued fuzzy $k$-ideal of $R$ but is not an interval valued fuzzy $h$-ideal of $R$.

Theorem 2.8. An interval valued fuzzy subset $\tilde{\lambda}=\left[\lambda^{-}, \lambda^{+}\right]$of a hemiring $R$ is an interval valued fuzzy left (resp. right) $k$-ideal of $R$ if and only if $\lambda^{-}$and $\lambda^{+}$are fuzzy left (resp. right) $k$-ideals of $R$.

Theorem 2.9. An interval valued fuzzy subset $\tilde{\lambda}=\left[\lambda^{-}, \lambda^{+}\right]$of a hemiring $R$ is an interval valued fuzzy left (resp. right) $h$-ideal of $R$ if and only if $\lambda^{-}$and $\lambda^{+}$are fuzzy left (resp. right) h-ideals of $R$.

Lemma 2.10. An interval valued fuzzy subset $\tilde{\lambda}$ of a hemiring $R$ is an interval valued fuzzy left (respectively right) ideal of $R$ if and only if $\tilde{\lambda}+\tilde{\lambda} \subseteq \tilde{\lambda}$ and $C_{R} \tilde{\lambda} \subseteq \tilde{\lambda}$ (respectively $\tilde{\lambda} C_{R} \subseteq \tilde{\lambda}$ ).

Proof. Proof is straightforward.

Theorem 2.11. If $\tilde{\lambda}$ and $\tilde{\mu}$ are interval valued fuzzy left (respectively right) ideals of $R$ then $\tilde{\lambda}+\tilde{\mu}$ and $\tilde{\lambda} \tilde{\mu}$ are interval valued fuzzy left (respectively right) ideals of $R$.

Proof. Proof is straightforward.

Theorem 2.12. Let $\tilde{\lambda}$ and $\tilde{\mu}$ be interval valued fuzzy left (resp. right) h-ideals of a hemiring $R$, then $\tilde{\lambda} \cap \tilde{\mu}$ is also an interval valued fuzzy left (resp. right) $h$-ideal of $R$.

Proof. Let $\tilde{\lambda}$ and $\tilde{\mu}$ be interval valued fuzzy left $h$-ideals of $R$, then $\tilde{\lambda} \cap \tilde{\mu}$ is an interval valued fuzzy left ideal of $R$. 
Let $a, b, x, y \in R$ such that $x+a+y=b+y$ then

$$
\begin{aligned}
(\tilde{\lambda} \cap \tilde{\mu})(x) & =\tilde{\lambda}(x) \wedge \tilde{\mu}(x) \\
& \geq\{\tilde{\lambda}(a) \wedge \tilde{\lambda}(b)\} \wedge\{\tilde{\mu}(a) \wedge \tilde{\mu}(b)\} \\
& =\{\tilde{\lambda}(a) \wedge \tilde{\mu}(a)\} \wedge\{\tilde{\lambda}(b) \wedge \tilde{\mu}(b)\} \\
& =(\tilde{\lambda} \cap \tilde{\mu})(a) \wedge(\tilde{\lambda} \cap \tilde{\mu})(b) .
\end{aligned}
$$

Thus $\tilde{\lambda} \cap \tilde{\mu}$ is an interval valued fuzzy left $h$-ideal of $R$.

Definition 14. Let $\tilde{\lambda}$ be an interval valued fuzzy subset of $R$ and $[\alpha, \beta] \in$ pounds then the level subset $U(\tilde{\lambda},[\alpha, \beta])$ of $R$ is defined as $U(\tilde{\lambda},[\alpha, \beta])=$ $\{x \in R: \tilde{\lambda}(x) \geq[\alpha, \beta]\}$.

Lemma 2.13. An interval valued fuzzy subset $\tilde{\lambda}$ of a hemiring $R$ is an interval valued fuzzy left (resp. right) $h$-ideal of $R$ if and only if each non-empty level subset of $R$ defined by $\tilde{\lambda}$ is a left (resp. right) $h$-ideal of $R$.

Theorem 2.14. Let $A$ be a non-empty subset of a hemiring $R$. Then the interval valued fuzzy subset $\tilde{\lambda}$ defined by

$$
\tilde{\lambda}(x)=\left\{\begin{array}{cc}
{[\alpha, \beta]} & \text { if } x \in A \\
{\left[\alpha_{o}, \beta_{o}\right]} & \text { if } x \notin A .
\end{array}\right.
$$

Where $\tilde{O} \leq\left[\alpha_{0}, \beta_{0}\right] \leq[\alpha, \beta] \leq \tilde{I}$ is an interval fuzzy left $h$-ideal of $R$ if and only if $A$ is a left $h$-ideal of $R$.

Proof. Let $\phi \neq A \subseteq R$ and $\tilde{\lambda}$ defined above is an interval valued fuzzy left $h$-ideal of $R$. Then for $x, y \in A$ we have

$$
\begin{aligned}
\tilde{\lambda}(x+y) & \geq \tilde{\lambda}(x) \wedge \tilde{\lambda}(y) \\
& =[\alpha, \beta] \wedge[\alpha, \beta]=[\alpha, \beta] .
\end{aligned}
$$

Thus $\tilde{\lambda}(x+y)=[\alpha, \beta]$. Hence $x+y \in A$.

Now let $x \in R$ and $y \in A$.

Then

$$
\tilde{\lambda}(x y) \geq \tilde{\lambda}(y)=[\alpha, \beta]
$$

Hence $\tilde{\lambda}(x y)=[\alpha, \beta]$. Thus $x y \in A$.

Let $a, x \in R$ and $y, z \in A$ such that $x+y+a=z+a$.

Then since $\tilde{\lambda}$ is an interval valued fuzzy left $h$-ideal of $R$, therefore

$$
\begin{aligned}
\tilde{\lambda}(x) & \geq \tilde{\lambda}(y) \wedge \tilde{\lambda}(z) \\
& =[\alpha, \beta] \wedge[\alpha, \beta]=[\alpha, \beta] .
\end{aligned}
$$

Hence $x \in A$, so $A$ is left $h$-ideal of $R$. 
Conversely, let $A$ be a left $h$-ideal of $R$ and $\tilde{\lambda}$ be an interval valued fuzzy subset of $R$, as defined in hypothesis. Then $\tilde{\lambda}$ is an interval valued fuzzy left ideal of $R$.

Now let $a, x, y, z \in R$ such that $x+y+a=z+a$. Then

CASE 1. When $y$ or $z \in R \backslash A$. Then

$$
\tilde{\lambda}(y)=\tilde{\lambda}(z)=\left[\alpha_{0}, \beta_{0}\right]
$$

$\Rightarrow \tilde{\lambda}(x) \geq \tilde{\lambda}(y) \wedge \tilde{\lambda}(z)$.

CASE 2 . When $y, z \in A$ then $x \in A$. Thus

$$
\tilde{\lambda}(x)=[\alpha, \beta] \geq\left[\alpha_{0}, \beta_{0}\right]=\tilde{\lambda}(y) \wedge \tilde{\lambda}(z) .
$$

Thus

$$
\tilde{\lambda}(x) \geq \tilde{\lambda}(y) \wedge \tilde{\lambda}(z) .
$$

Hence $\tilde{\lambda}$ is an interval valued fuzzy left $h$-ideal of $R$.

Lemma 2.15. If $\tilde{\lambda}$ and $\tilde{\mu}$ are interval valued fuzzy right h-ideal and interval valued fuzzy left $h$-ideal of a hemiring $R$, respectively. Then

$$
\tilde{\lambda} \odot \tilde{\mu} \subseteq \tilde{\lambda} \cap \tilde{\mu} .
$$

Proof. Let $x \in R$, If $x$ cannot be expressed as $x+\sum_{i=1}^{n} a_{i} b_{i}+z=\Sigma_{j=1}^{m} c_{j} d_{j}+z$ for any $a_{i}, b_{i}, c_{j}, d_{j}, z \in R$ then

$$
(\tilde{\lambda} \odot \tilde{\mu})(x)=\tilde{O} \leq \tilde{\lambda}(x) \wedge \tilde{\mu}(x)=(\tilde{\lambda} \cap \tilde{\mu})(x) .
$$

Otherwise, since $\tilde{\lambda}$ and $\tilde{\mu}$ are interval valued fuzzy $h$-ideals so

$$
\tilde{\lambda}(x) \geq \tilde{\lambda}\left(\sum_{i=1}^{n} a_{i} b_{i}\right) \wedge \tilde{\lambda}\left(\sum_{j=1}^{m} c_{j} d_{j}\right)
$$

and

$$
\tilde{\mu}(x) \geq \tilde{\mu}\left(\sum_{i=1}^{n} a_{i} b_{i}\right) \wedge \tilde{\mu}\left(\sum_{j=1}^{m} c_{j} d_{j}\right) .
$$

Now

$$
\begin{aligned}
& (\tilde{\lambda} \cap \tilde{\mu})(x) \\
= & \tilde{\lambda}(x) \wedge \tilde{\mu}(x) \\
\geq & \tilde{\lambda}\left(\sum_{i=1}^{n} a_{i} b_{i}\right) \wedge \tilde{\lambda}\left(\sum_{j=1}^{m} c_{j} d_{j}\right) \wedge \tilde{\mu}\left(\sum_{i=1}^{n} a_{i} b_{i}\right) \wedge \tilde{\mu}\left(\sum_{j=1}^{m} c_{j} d_{j}\right) \\
\geq & \wedge_{i, j}\left\{\tilde{\lambda}\left(a_{i} b_{i}\right) \wedge \tilde{\lambda}\left(c_{j} d_{j}\right) \wedge \tilde{\mu}\left(a_{i} b_{i}\right) \wedge \tilde{\mu}\left(c_{j} d_{j}\right)\right\} \\
\geq & \wedge_{i, j}\left\{\tilde{\lambda}\left(a_{i}\right) \wedge \tilde{\lambda}\left(c_{j}\right) \wedge \tilde{\mu}(b i) \wedge \tilde{\mu}\left(d_{j}\right)\right\} .
\end{aligned}
$$

Since above expression holds for any $a_{i}, b_{i}, c_{j}, d_{j} \in R$ and for all $i, j$ therefore 


$$
\begin{aligned}
& (\tilde{\lambda} \cap \tilde{\mu})(x)
\end{aligned}
$$

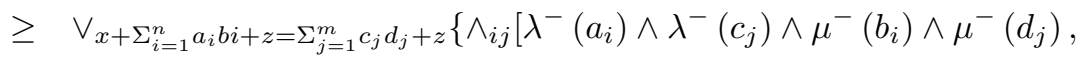

$$
\begin{aligned}
& \left.\left.\lambda^{+}\left(a_{i}\right) \wedge \lambda^{+}\left(c_{j}\right) \wedge \mu^{+}\left(b_{i}\right) \wedge \mu^{+}\left(d_{j}\right)\right]\right\} \\
& =(\tilde{\lambda} \odot \tilde{\mu})(x) \\
& \Rightarrow
\end{aligned}
$$

$$
\tilde{\lambda} \odot \tilde{\mu} \subseteq \tilde{\lambda} \cap \tilde{\mu} .
$$

Lemma 2.16. Let $R$ be a hemiring and $\tilde{\lambda}, \tilde{\mu}, \tilde{\nu}, \tilde{\varpi}$ be any interval valued fuzzy subsets in $R$ such that $\tilde{\lambda} \subseteq \tilde{\nu}$ and $\tilde{\mu} \subseteq \tilde{\varpi}$ then

$$
\tilde{\lambda} \odot \tilde{\mu} \subseteq \tilde{\nu} \odot \tilde{\varpi} .
$$

Proof. If $x$ can not be written in the form $x+\sum_{i=1}^{n} a_{i} b_{i}+z=\sum_{i=1}^{m} c_{j} d_{j}+z$ for any $a_{i}, b_{i}, c_{j}, d_{j}, z \in R$ then

$$
(\tilde{\lambda} \odot \tilde{\mu})(x)=\tilde{O}=(\nu \odot \tilde{\varpi})(x) .
$$

Otherwise since $\tilde{\lambda} \subseteq \tilde{\nu}$ and $\tilde{\mu} \subseteq \tilde{\varpi}$ so

$$
\begin{array}{llll}
\lambda^{-} & \subseteq & \nu^{-} & \lambda^{+} \subseteq \nu^{+} \\
\mu^{-} & \subseteq & \varpi^{-} & \mu^{+} \subseteq \varpi^{+}
\end{array}
$$

and hence for all $x \in R$

$$
\begin{aligned}
& (\tilde{\lambda} \odot \tilde{\mu})(x) \\
= & \vee_{x+\Sigma_{i=1}^{n} a_{i} b_{i}+z=\Sigma_{j=1}^{m} c_{j} d_{j}+z}\left\{\wedge _ { i , j } \left[\lambda^{-}\left(a_{i}\right) \wedge \lambda^{-}\left(c_{j}\right) \wedge \mu^{-}\left(b_{i}\right) \wedge \mu^{-}\left(d_{j}\right),\right.\right. \\
\left.\left.\lambda^{+}\left(a_{i}\right) \wedge \lambda^{+}\left(c_{j}\right) \wedge \mu^{+}\left(b_{i}\right) \wedge \mu^{+}\left(d_{j}\right)\right]\right\} & \\
\leq \quad & \vee_{x+\Sigma_{i=1}^{n} a_{i} b_{i}+z=\Sigma_{j=1}^{m} c_{j} d_{j}+z\left\{\wedge _ { i , j } \left[\nu^{-}\left(a_{i}\right) \wedge \nu^{-}\left(c_{j}\right) \wedge \varpi^{-}\left(b_{i}\right) \wedge \varpi^{-}\left(d_{j}\right),\right.\right.}^{\left.\left.\nu^{+}\left(a_{i}\right) \wedge \nu^{+}\left(c_{j}\right) \wedge \varpi^{+}\left(b_{i}\right) \wedge \varpi^{+}\left(d_{j}\right)\right]\right\}} \\
= & (\tilde{\nu} \odot \tilde{\varpi})(x) \quad \tilde{\lambda} \odot \tilde{\mu} \subseteq \tilde{\nu} \odot \tilde{\varpi} .
\end{aligned}
$$

Theorem 2.17. An interval valued fuzzy subset $\tilde{\lambda}$ of a hemiring $R$ is an interval valued fuzzy left (respectively right) $h$-ideal of $R$ if and only if for all $x, y, a, b \in R$, we have

(i) $\tilde{\lambda}(x+y) \geq \tilde{\lambda}(x) \wedge \tilde{\lambda}(y)$ 
(ii) $\tilde{C}_{R} \odot \tilde{\lambda} \subseteq \tilde{\lambda}\left(\right.$ respectively $\left.\tilde{\lambda} \odot \tilde{C}_{R} \subseteq \tilde{\lambda}\right)$,

(iii) $x+a+y=b+y \Rightarrow \tilde{\lambda}(x) \geq \tilde{\lambda}(a) \wedge \tilde{\lambda}(b)$.

Proof. Let $\tilde{\lambda}$ be an interval valued fuzzy left $h$-ideal of $R$, then by definition $(i)$ and (iii) are true. Now let $x \in R$. If $x$ cannot be written as $x+\sum_{i=1}^{n} a_{i} b_{i}+y=$ $\sum_{j=1}^{m} a_{j}^{\prime} b_{j}^{\prime}+y$ for any $a_{i}, a_{j}^{\prime}, b_{i}, b_{j}^{\prime}, y \in R$, then

$$
\left(\tilde{C}_{R} \odot \tilde{\lambda}\right)(x)=\tilde{O} \leq \tilde{\lambda}(x) .
$$

Otherwise

$$
\begin{aligned}
\left(\tilde{C}_{R} \odot \tilde{\lambda}\right)(x) & =\vee_{x+\Sigma_{i=1}^{n} a_{i} b_{i}+y=\sum_{j=1}^{m} a_{j}^{\prime} b_{j}^{\prime}+y}\left\{\wedge_{i, j}\left[\begin{array}{c}
\lambda^{-}\left(b_{i}\right) \wedge \lambda^{-}\left(b_{j}^{\prime}\right), \\
\lambda^{+}\left(b_{i}\right) \wedge \lambda^{+}\left(b_{j}^{\prime}\right)
\end{array}\right]\right\} \\
& \leq \vee_{x+\Sigma_{i=1}^{n} a_{i} b_{i}+y=\Sigma_{j=1}^{m} a_{j}^{\prime} b_{j}^{\prime}+y}\left\{\wedge_{i, j}\left[\begin{array}{c}
\lambda^{-}\left(a_{i} b_{i}\right) \wedge \lambda^{-}\left(a_{j}^{\prime} b_{j}^{\prime}\right), \\
\lambda^{+}\left(a_{i} b_{i}\right) \wedge \lambda^{+}\left(a_{j}^{\prime} b_{j}^{\prime}\right)
\end{array}\right]\right\} \\
& \leq \vee_{x+\sum_{i=1}^{n} a_{i} b_{i}+y=\Sigma_{j=1}^{m} a_{j}^{\prime} b_{j}^{\prime}+y}\left[\begin{array}{c}
\lambda^{-}\left(\sum_{i=1}^{n} a_{i} b_{i}\right) \wedge \lambda^{-}\left(\sum_{j=1}^{m} a_{j}^{\prime} b_{j}^{\prime}\right), \\
\lambda^{+}\left(\sum_{i=1}^{n} a_{i} b_{i}\right) \wedge \lambda^{+}\left(\sum_{j=1}^{m} a_{j}^{\prime} b_{j}^{\prime}\right)
\end{array}\right] \\
= & \vee_{x+\sum_{i=1}^{n} a_{i} b_{i}+y=\Sigma_{j=1}^{m} a_{j}^{\prime} b_{j}^{\prime}+y}\left(\tilde{\lambda}\left(\sum_{i=1}^{n} a_{i} b_{i}\right) \wedge \tilde{\lambda}\left(\sum_{j=1}^{m} a_{j}^{\prime} b_{j}^{\prime}\right)\right) \\
\leq & \vee \tilde{\lambda}(x)) \quad \because \tilde{\lambda} \text { is interval valued fuzzy left } h-\text { ideal } \\
= & \tilde{\lambda}(x) .
\end{aligned}
$$

Hence $\tilde{C}_{R} \odot \tilde{\lambda} \subseteq \tilde{\lambda}$. Conversely, assume that $(i),(i i),(i i i)$ hold for an interval valued fuzzy subset $\tilde{\lambda}$ of $R$, then to prove that $\tilde{\lambda}$ is an interval valued fuzzy left $h$-ideal of $R$,we only have to show that $\tilde{\lambda}(x y) \geq \tilde{\lambda}(y)$ for all $x, y \in R$. So let $x, y \in R$, then by $(i i)$

$$
\begin{aligned}
& \tilde{\lambda}(x y) \geq\left(\tilde{C}_{R} \odot \tilde{\lambda}\right)(x y) \\
& =\vee_{x y+\Sigma_{i=1}^{n} a_{i} b_{i}+z=\Sigma_{j=1}^{m} a_{j}^{\prime} b_{j}^{\prime}+z}\left\{\wedge_{i, j}\left[\lambda^{-}\left(b_{i}\right) \wedge \lambda^{-}\left(b_{j}^{\prime}\right), \lambda^{+}\left(b_{i}\right) \wedge \lambda^{+}\left(b_{j}^{\prime}\right)\right]\right\} \\
& \geq\left[\lambda^{-}(y) \wedge \lambda^{-}(y), \lambda^{+}(y) \wedge \lambda^{+}(y)\right] \quad \text { because } x y+0 y+0=x y+0 \\
& =\tilde{\lambda}(y) \text {. }
\end{aligned}
$$

Therefore $\tilde{\lambda}(x y) \geq \tilde{\lambda}(y)$ for all $x, y \in R$.

Lemma 2.18. Let $R$ be a hemiring and $A, B \subseteq R$ then

$$
\tilde{C}_{A} \odot \tilde{C}_{B}=\tilde{C}_{\overline{A B}}
$$


Proof. Let $x \in R$. If $x \in \overline{A B}$ then $\tilde{C}_{\overline{A B}}(x)=\tilde{I}$ and $x+\sum_{i=1}^{n} a_{i} b_{i}+z=\sum_{j=1}^{m} a_{j}^{\prime} b_{j}^{\prime}+z$ for some $a_{i}, a_{j}^{\prime} \in A$ and $b_{i}, b_{j}^{\prime} \in B$ and $z \in R$.Thus

$$
\tilde{C}_{A}\left(a_{i}\right)=\tilde{C}_{A}\left(a_{j}^{\prime}\right)=\tilde{C}_{B}\left(b_{i}\right)=\tilde{C}_{B}\left(b_{j}^{\prime}\right)=\tilde{I}
$$

and hence

$$
\begin{aligned}
& \left(\tilde{C}_{A} \odot \tilde{C}_{B}\right)(x) \\
& =\vee_{x+\Sigma_{i=1}^{n} a_{i} b_{i}+z=\Sigma_{j=1}^{m} a_{j}^{\prime} b_{j}^{\prime}+z}\left\{\wedge_{i, j}\left[\begin{array}{c}
C_{A}^{-}\left(a_{i}\right) \wedge C_{A}^{-}\left(a_{j}^{\prime}\right) \wedge C_{B}^{-}\left(b_{i}\right) \wedge C_{B}^{-}\left(b_{j}^{\prime}\right), \\
C_{A}^{+}\left(a_{i}\right) \wedge C_{A}^{+}\left(a_{j}^{\prime}\right) \wedge C_{B}^{+}\left(b_{i}\right) \wedge C_{B}^{+}\left(b_{j}^{\prime}\right)
\end{array}\right]\right\}
\end{aligned}
$$

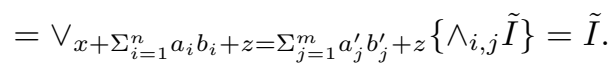

Therefore whenever $x \in \overline{A B}$ then

$$
\left(\tilde{C}_{A} \odot \tilde{C}_{B}\right)(x)=\left(\tilde{C}_{\overline{A B}}\right)(x)=\tilde{I} .
$$

And if $x \notin \overline{A B}$ then $\tilde{C}_{\overline{A B}}(x)=\tilde{O}$.

If possible, let $\left(\tilde{C}_{A} \odot \tilde{C}_{B}\right)(x) \neq \tilde{O}$ then $\vee_{x+\Sigma_{i=1}^{n} a_{i} b_{i}+z=\Sigma_{j=1}^{m} a_{j}^{\prime} b_{j}^{\prime}+z}\left\{\wedge_{i, j}\left[\begin{array}{c}C_{A}^{-}\left(a_{i}\right) \wedge C_{A}^{-}\left(a_{j}^{\prime}\right) \wedge \\ C_{B}^{-}\left(b_{i}\right) \wedge C_{B}^{-}\left(b_{j}^{\prime}\right), \\ C_{A}^{+}\left(a_{i}\right) \wedge C_{A}^{+}\left(a_{j}^{\prime}\right) \wedge \\ C_{B}^{+}\left(b_{i}\right) \wedge C_{B}^{+}\left(b_{j}^{\prime}\right)\end{array}\right]\right\} \neq[0,0]$.

Therefore there exist $p_{i}, q_{i}, p_{j}^{\prime}, q_{j}^{\prime} \in R$ such that

$$
x+\sum_{i=1}^{n} p_{i} q_{i}+z=\sum_{j=1}^{m} p_{j}^{\prime} q_{j}^{\prime}+z
$$

and

$$
\wedge_{i, j}\left[\begin{array}{c}
C_{A}^{-}\left(p_{i}\right) \wedge C_{A}^{-}\left(p_{j}^{\prime}\right) \wedge C_{B}^{-}\left(q_{i}\right) \wedge C_{B}^{-}\left(q_{j}^{\prime}\right), \\
C_{A}^{+}\left(p_{i}\right) \wedge C_{A}^{+}\left(p_{j}^{\prime}\right) \wedge C_{B}^{+}\left(q_{i}\right) \wedge C_{B}^{+}\left(q_{j}^{\prime}\right)
\end{array}\right] \neq[0,0] .
$$

Then obviously for all $i$ and $j$

$$
C_{A}^{-}\left(p_{i}\right)=C_{A}^{-}\left(p_{j}^{\prime}\right)=C_{B}^{-}\left(q_{i}\right)=C_{B}^{-}\left(q_{j}^{\prime}\right)=1
$$

and

$$
C_{A}^{+}\left(p_{i}\right)=C_{A}^{+}\left(p_{j}^{\prime}\right)=C_{B}^{+}\left(q_{i}\right)=C_{B}^{+}\left(q_{j}^{\prime}\right)=1
$$

$\Rightarrow \quad$ for all $i$ and $j$

$$
\tilde{C}_{A}\left(p_{i}\right)=\tilde{C}_{A}\left(q_{i}\right)=\tilde{I}
$$

and

$$
\tilde{C}_{A}\left(p_{j}^{\prime}\right)=\tilde{C}_{A}\left(q_{j}^{\prime}\right)=\tilde{I}
$$

$\Rightarrow$

$$
p_{i} \in A, q_{i} \in B \quad \forall i
$$

and

$$
p_{j}^{\prime} \in A, q_{j}^{\prime} \in B \quad \forall j
$$

$\Rightarrow$

$$
x \in A B
$$


which contradicts $\tilde{C}_{\overline{A B}}(x)=\tilde{O}$. Therefore whenever $x \notin \overline{A B}$ then again we have

$$
\left(\tilde{C}_{A} \odot \tilde{C}_{B}\right)(x)=\tilde{O}=\tilde{C}_{\overline{A B}}(x)
$$

Hence proved that

$$
\tilde{C}_{A} \odot \tilde{C}_{B}=\tilde{C}_{\overline{A B}}
$$

Theorem 2.19. A hemiring $R$ is h-hemiregular if and only if for any interval valued fuzzy right $h$-ideal $\tilde{\lambda}$ and interval valued fuzzy left $h$-ideal $\tilde{\mu}$ of $R$, we have

$$
\tilde{\lambda} \odot \tilde{\mu}=\tilde{\lambda} \cap \tilde{\mu} .
$$

Proof. By Lemma 2.15, $\tilde{\lambda} \odot \tilde{\mu} \subseteq \tilde{\lambda} \cap \tilde{\mu}$. Now for reverse containment, since $R$ is $h$-hemiregular so for all $a \in \bar{R}$, there exist $x_{1}, x_{2}, y \in R \quad$ such that

$$
a+a x_{1} a+y=a x_{2} a+y
$$

Now

$$
\begin{aligned}
& (\tilde{\lambda} \odot \tilde{\mu})(a) \\
& =\vee_{a+\Sigma_{i=1}^{n} a_{i} b_{i}+y=\Sigma_{j=1}^{m} c_{j} d_{j}+y}\left\{\wedge_{i j}\left[\begin{array}{c}
\lambda^{-}\left(a_{i}\right) \wedge \lambda^{-}\left(c_{j}\right) \wedge \mu^{-}\left(b_{i}\right) \wedge \mu^{-}\left(d_{j}\right), \\
\lambda^{+}\left(a_{i}\right) \wedge \lambda^{+}\left(c_{j}\right) \wedge \mu^{+}\left(b_{i}\right) \wedge \mu^{+}\left(d_{j}\right)
\end{array}\right]\right\} \\
& \geq\left[\lambda^{-}\left(a x_{1}\right) \wedge \lambda^{-}\left(a x_{2}\right) \wedge \mu^{-}(a) \wedge \mu^{-}(a), \mu^{+}(a) \wedge \lambda^{+}\left(a x_{1}\right) \wedge \lambda^{+}\left(a x_{2}\right) \wedge \mu^{+}(a)\right] \\
& \geq\left[\lambda^{-}(a) \wedge \lambda^{-}(a) \wedge \mu^{-}(a) \wedge \mu^{-}(a), \lambda^{+}(a) \wedge \lambda^{+}(a) \wedge \mu^{+}(a) \wedge \mu^{+}(a)\right. \\
& =\left[\lambda^{-}(a) \wedge \mu^{-}(a), \lambda^{+}(a) \wedge \mu^{+}(a)\right]=\tilde{\lambda}(a) \wedge \tilde{\mu}(a)=(\tilde{\lambda} \cap \tilde{\mu})(a) \text {. }
\end{aligned}
$$

Thus

$$
\tilde{\lambda} \cap \tilde{\mu} \subseteq \tilde{\lambda} \odot \tilde{\mu} .
$$

Hence

$$
\tilde{\lambda} \odot \tilde{\mu}=\tilde{\lambda} \cap \tilde{\mu} .
$$

Conversely, let $A$ and $B$ be right and left $h$-ideals of $R$ respectively, then by Lemma 2.7 their interval valued characteristic functions $\tilde{C}_{A}$ and $\tilde{C}_{B}$ are also interval valued fuzzy right and interval valued fuzzy left $h$-ideals of $R$ respectively. Then by hypothesis

$$
\tilde{C}_{\overline{A B}}=\tilde{C}_{A} \odot \tilde{C}_{B}=\tilde{C}_{A} \cap \tilde{C}_{B}=\tilde{C}_{A \cap B} .
$$

Thus $\overline{A B}=A \cap B$. Hence by Lemma $2.4, R$ is $h$-hemiregular. 


\section{References}

[1] A. W. Aho and J. D. Ullman, Introduction to Automata Theory, Languages and Computation, Addison Wesley, Reading, MA, 1979.

[2] J. Ahsan, K. Saifullah and M. F. Khan, Fuzzy Semirings, Fuzzy Sets Syst. 60 (1993), 309-320.

[3] J. Ahsan, Semirings characterized by their fuzzy ideals, J. Fuzzy Math. 6 (1998), 181192.

[4] L. B. Beasley and N. G. Pullman, Operators that preserves semiring matrix functions, Linear Algebra Appl. 99 (1988), 199-216.

[5] L. B. Beasley and N. G. Pullman, Linear operators strongly preserving idempotent matrices over semirings, Linear Algebra Appl. 160 (1992), 217-229

[6] R. Biswas, Rosenfeld's fuzzy subgroups with intervalued membership function, Fuzzy sets and systems 63 (1994), 87-90.

[7] W. A. Dudek, M. Shabir and R. Anjum, Characterizations of Hemirings by their hideals, Comp. and Maths. with Appl. 59 (2010), 3167-3179.

[8] S. Ghosh, Matrices over semirings, Inform. Sci. 90 (1996), 221-230.

[9] K. Glazek, A guide to litrature on semirings and their applications in mathematics and information sciences: with complete bibliography, Kluwer Acad. Publ. Nederland, 2002.

[10] J. S. Golan, Semirings and their applications, Kluwer Acad. Publ. 1999.

[11] U. Hebisch and H. J. Weinert, Semirings: Algebraic Theory and Applications in the Computer Science, World Scientific, 1998.

[12] M. Henriksen, Ideals in semirings with commutative addition, Amer. Math. Soc. Notices 6 (1958), 321.

[13] K. Iizuka, On Jacobson radical of a semiring, Tohoku Math. J. 11 (1959), 409-421.

[14] D. R. La Torre, On h-ideals and k-ideals in hemirings, Publ. Math. Debrecen 12 (1965), 219-226.

[15] X. P. Li and G. J. Wang, The $S_{H}$ interval valued fuzzy subgroups, Fuzzy sets and systems 112 (2000), 319-325.

[16] J. N. Mordeson and D. S. Malik, Fuzzy Automata and Languages, Theory and Applications, Computational Mathematics Series, Chapman and Hall/CRC, Boca Raton 2002.

[17] A. Rosenfeld, Fuzzy groups, J. Math. Anal. Appl. 35 (1971), 512-517.

[18] M. Shabir and T. Mahmood, Characterizations of Hemirings by Interval Valued fuzzy ideals, Quasigroups and Related Systems 19 (2011), 101-113.

[19] G. Sun, Y. Yin and Y. Li, Interval valued fuzzy h-ideals of hemirings, Int. Math. Forum 5 (2010), 545-556.

[20] W. Wechler, The concept of fuzziness in automata and language theory, Akademic ver$\log$, Berlin, 1978.

[21] Xueling M. A. and J. Zhan, On fuzzy h-ideals of hemirings, J. Syst. Sci. and Complexity 20 (2007), 470-478.

[22] Y. Q. Yin and H. Li, The charatecrizations of h-hemiregular hemirings and h-intrahemiregular hemirings, Inform. Sci. 178 (2008), 3451-3464.

[23] H. S. Vandiver, Note on a simple type of algebra in which cancellation law of addition does not hold, Bull. Amer. Math. Soc. 40 (1934), 914-920

[24] L. A. Zadeh, Fuzzy Sets, Information and Control 8 (1965), 338-353. 
Muhammad Shabir

Department of Mathematics, Quaid-I-Azam University, Islamabad, Pakistan

E-mail address: mshabirbhatti@yahoo.co.uk

NOSHIN MALIK

Department of Mathematics, Quaid-I-Azam University, Islamabad, Pakistan

TAHIR MAHMOOD

Department of Mathematics, International Islamic University, Islamabad, PakISTAN

E-mail address: tahirbakhat@yahoo.com 